\title{
Establecimiento y mantenimiento de una colonia de Aedes taeniorhynchus, Wiedemann, 1821 (Díptera: Cullicidae) cepa Barranquilla, Colombia.
}

\author{
Bello F.J. ${ }^{1}$, Olano V.A. ${ }^{2}$, Morales A. ${ }^{3}$, Cassaleth E. ${ }^{4}$, Giraldo L. ${ }^{4}$ Hernánder $C .{ }^{4}$
}

\begin{abstract}
Resumen
Se estableció una colonia del mosquito Aedes taeniorhynchus, eficientevector del virus de la encefalitis equina venezolana, tipo epidemo-epizoótico, a partir deformas adultas, recolectados en regiones cercanas a Barranquilla, Atlántico (Colombia). Se estudiaron algunos aspectos de la biología de este insecto (ciclo de vida y longevidad), elaboración de tablas de vidae igualmente se maximizaron algunos parámetrosfísicos, ambientales y nutricionales que garantizarán el mantenimiento y la continuidad de la especie en condiciones de laboratorio. Esta colonia fue establecida con el objeto de proporcionar la materia prima biológica para los explantes de tejidos embrionarios que se han venido utilizando para establecer líneas celulares de crecimiento continuo.
\end{abstract}

\begin{abstract}
Summary
Acolony of Aedes taeniorhynchus, an efficientvectorof Venezuelan Equine Encephalitis, epidemo-epizootic type, was established. It was started by using adult forms, collected from regions close to the Atlantic coast town of Barranquilla (Colombia)

Some biological aspects of this insect were studied, including life cycles and longevity. Actuarialtables were preparedand likewise, somephysical, environmental and nutritional parameters were established that guaranteed the maintainance and continuity of the species in laboratory conditions. The objective of this colony was to provide biological raw material for embryonic tissue explants that have been used to establish cell lines of continued growth.
\end{abstract}

El mosquito Aedes taeniorhynchus es un vector eficiente del virus de la encefalitis equina venezolana, (EEV) tipo epidemo-epizoótico. Este insecto presenta una vasta distribución continental, siendo encontrado desdelas regionescosteras dellado del Atlántico, extendiéndosedesdeMassachusetts, en los E.U.A., hasta Santa Catalina al

'M.Sc. Investigador, Universidad de La Sarle

2 Biólogo. Grupo de Entomología, Instituto Nacional de Salud

3 M.Sc., Jefe, Grupo de Entornología. Instituto Nacional de Salud

4 Estudiantes de pregrado, Universidad de La Salle surdel Brasily dellado del Pacífico desde California en los E.U.A. hasta el Perú. El mosquito es particularmente abundante en el litoral sureste de los E.U.A. Su distribución incluye también las Antillas y las islas Galápagos (3). En Colombiase le ha encontrado en las costas del Atlántico y del Pacifico, pero en 1985, fue detectado en el interior del país en Ambalema, Tolima (8).

El virus de la encefalitis equinavenezolana, está ampliamente distribuido en el continente americano:Perú, Ecuador, Colombia, Venezuela, Costa Rica, Honduras, El Salvador, Guatemala, México y E.U.A. (1). Lainvestigación de laecologíade 
los arbovirus en América tropical durante las tres décadas pasadas ha dado por resultado la identificación de numerosos subtipos antigénicos del virus de la encefalitis equina venezolana que difierenen patogenicidad para equinos y posiblemente para humanos y con ciclos de mantenimiento y trasmisión distintos. Tomados en conjunto, los virus de la EEV han matado más equinos y producido más enfermedades humanas en el hemisferio occidental que cualquiera de los otros arbovirus (6).

Trabajos relativamente recientes sobre el comportamiento de Aedes taeniorhynchus en zonas tropicales, muestran que el máximo de las densidades de población coinciden con el de las mayores precipitaciones, mientras que la temperatura y la humedad relativa no tienen influencias significativas sobre las poblaciones de esta especie (4).

Esta especie de mosquitos ha sido colonizado en diferentes instituciones de los E.U.A. (5). Con el establecimiento y mantenimiento de una colonia de Aedes taeniorhynchus, cepa Barranquilla, Colombia, en principio, se busca adquirir los conocimientos esenciales sobre algunos aspectos de la biología de estos mosquitos (ciclo de vida y longevidad), elaboración de tablas de vida y evaluación de algunos parámetros que maximizarán la continuidad de la especie en condiciones de laboratorio.

Fundamentalmente la colonia fue establecida con el fin de proporcionar la materia prima biológica para los explantes de tejidos embrionarios con miras a obtener cultivos primarios y lograr líneas celulares de crecimiento continuo.

\section{Materiales y métodos}

- Ubicación del área. Las formas adultas de Aedes taeniorhynchus se recolectaron en su hábitat natural, en regiones cercanas a Barranquilla, Atlántico (Colombia), en cinco localidades: Barrio Palermo en el kilómetro cero, finca El Establo, en el kilómetro 7, finca Los Hermanos en el kilómetro 11, Instituto Nacional para el Desarrollo de los Recursos Naturales y del Ambiente, INDERENA, muni- cipio de Sitio Nuevo, Magdalena y Puerto Mocho, Atlántico.

- Captura de ejemplares. Los adultos se recolectaron en la superficies donde usualmente se encuentran reposando (estructuras que conforman el establo, orillas de un caño y vegetaciones del manglar) y sobre algunos animales como vaca y burro.

Se capturaron con un aspirador de boca y se guardaron en vasos de cartón. Estos a su vez se colocaron en el interior de una nevera de icopor para ser transportados y posteriormente ser identificados.

Las capturas se realizaron durante la noche (de 6:00 p.m. a 9:00 p.m.) y en la madrugada (de 4:00 a.m. a 7:00 a.m.) los días 1, 2 y 3 de diciembre de 1992.

- Clasificación del material y traslado al laboratorio. La identificación se realizó teniendo en cuenta las características morfológicas (pigmentación de las patas, color y tamaño del mosquito). Los mosquitos Aedes taeniorhynchus identificados fueron depositados en jaulas y éstas a su vez dentro de cajas de icopor para su traslado al insectario del INS en Santafé de Bogotá, D.C., el cual poseía una temperatura y humedad relativa promedio de $27^{\circ} \mathrm{C}$ y $80 \%$, respectivamente, condiciones adecuadas para el mantenimiento de la especie.

- Manejo del ciclo de vida. Los adultos fueron colocados en jaulas metálicas Gerberg. Para la oviposición se dejó en cada jaula durante 10 días un par de tazas plásticas llenas hasta la mitad con tierra humedecida. Transcurrido este tiempo, las tazas se retiraban de la jaula y se observaban al esteroscopio para verificar si había buena postura, en cuyo caso se retiraban y reemplazaban por otras. Al completar los huevos el tiempo de incubación, se inundaban las tazas en cápsulas de porcelana para que eclosionaran las larvas. Estas podían permanecer en las tazas hasta completar su ciclo o se transferían a bandejas esmaltadas. Las pupas que resultaban al fina- 
lizar el desarrollo larval se pasaban a tazas con agua, las cuales se tapaban hasta que emergían los adultos, que se dejaban libres al interior de las jaulas.

- Mantenimiento de la colonia. Para el mantenimiento normal de la colonia se efectuaba el aseo y limpieza diariamente de las jaulas, bandejas y utensilios empleados, igualmente se tenían en cuenta medidas de seguridad para evitar la contaminación de la colonia con otros mosquitos.

La alimentación que se administraba a las larvas consistía en Ken-L molido (alimento para perros). Los adultos hembras se alimentaban con sangre de curí. A los adultos machos y hembras se les mantenía permanentemente una chupa (bola de algodón envuelta en un pedazo de gasa) impregnada de una solución azucarada, sostenida con un alambre en la parte superior interna de la jaula.

- Observación y registro. Durante todo el proceso, se mantuvo una rigurosa observación diaria de cada una de las etapas del ciclo respectivo.
- Montaje, conservación e identificación de larvas $y$ adultos. A una muestra significativa de material larval y adultos, se les hizo el procedimiento de montaje permanente y conservación (2); para la identificación taxonómica se utilizaron las claves de Nielsen (7) y Tinker (10)

\section{Resultados}

Durante el establecimiento y mantenimiento de la colonia, se estudiaron seis generaciones y de cada una de ellas se registró el número de pupas vivas y muertas, con lo cual se determinó el porcentaje de emergencia de adultos. Del 100\% de las pupas, emergen un promedio de $95,68 \%$ de adultos (tabla 1).

El tiempo promedio de eclosión de la larva fue 10,73 minutos.

En la tabla 2 se muestran los valores correspondientes a la duración promedio de cada uno de los estadios del ciclo de vida del mosquito, a saber: períodos de incubación 4,5 días, de larva a pupa 8,6 días y de pupa a adulto 1,7 días. El promedio de vida de la hembra adulta fue de 15,9 días y del macho de 11,8 días.

Tabla 1. Valores de supervivencia y duración del mosquito Aedes taeniorhychus bajo condiciones de laboratorio en las seis generaciones estudiadas.

\begin{tabular}{|c|c|c|c|c|c|c|c|c|}
\hline Generación & $\begin{array}{c}\text { Número } \\
\text { de tazas } \\
\text { con postura }\end{array}$ & $\begin{array}{l}\text { Número } \\
\text { de tazas } \\
\text { inundadas }\end{array}$ & $\begin{array}{l}\text { Número de } \\
\text { pupas vivas }\end{array}$ & $\begin{array}{l}\text { Número de } \\
\text { pupas muertas }\end{array}$ & $\begin{array}{l}\text { Porcentaje de } \\
\text { s emergencia }\end{array}$ & $\begin{array}{l}\text { Tiempo de } \\
\text { eclosión }\end{array}$ & $\begin{array}{c}\text { Duración } \\
\text { por días } \\
\text { larva-pupa }\end{array}$ & $\begin{array}{c}\text { Duración } \\
\text { por días } \\
\text { pupa-adulto }\end{array}$ \\
\hline \multirow[t]{2}{*}{$\mathrm{F} 1$} & 5 & 5 & 1,381 & 89 & 93,55 & 12,75 & 8,60 & 1,40 \\
\hline & & & & & & 4,99 & 1,14 & 0,55 \\
\hline \multirow[t]{2}{*}{$\mathrm{F} 2$} & 10 & 9 & 4,932 & 246 & 95,01 & 10,67 & 9,33 & 1,67 \\
\hline & & & & & & 2,34 & 1,50 & 0,50 \\
\hline \multirow[t]{2}{*}{ F3 } & 16 & 5 & 2,748 & 86 & 96,87 & 12,33 & 8,60 & 2,00 \\
\hline & & & & & & 3,78 & 0,89 & 0,00 \\
\hline \multirow[t]{2}{*}{$\mathrm{F} 4$} & 13 & 4 & 1,532 & 15 & 99,02 & 10,50 & 7,50 & 2,00 \\
\hline & & & & & & 2,08 & 1,91 & 0,00 \\
\hline \multirow[t]{2}{*}{$\mathrm{F} 5$} & 5 & 3 & 3,348 & 78 & 97,67 & 5,33 & 8,33 & 2,00 \\
\hline & & & & & & 0,58 & 1,15 & 0,00 \\
\hline \multirow[t]{2}{*}{ F6 } & 6 & 2 & 150 & 12 & 92,00 & 9,00 & 9,00 & 2,00 \\
\hline & & & & & & 1,41 & 1,41 & 0,00 \\
\hline
\end{tabular}


La duración total del ciclo de vida para las hembras es de 30,8 días (figura 1) y para los ma-chos de 26,7 días (figura 2).

En los cálculos para las tablas de vida se usó una cohorte de 200 mosquitos adultos. Se comenzó con 100 machos y 100 hembras, contando diariamente el número de individuos que sobreviven (9). La tabla de vida para los machos es la 3 y para las hembras la 4 . Se muestra también la curva de supervivencia para esta cohorte en la figura 3.

El tiempo promedio de vida para hembras criadas en viales fue de 19,7 días. Para obtener tales datos, se comenzó con 10 hembras nacidas en el mismo día, a cada una de ellas se les registraba el día de su muerte y el número de días que duraba viva.

Tabla 2. Duración de los estadios de desarrollo de! mosquito Aedes taeniorhynchus en condiciones de laboratorio.

\begin{tabular}{lcc}
\hline Estadio & Duración (días) & Duración estándar \\
\hline Huevo & 4,50 & 0,71 \\
Larva & 8,68 & 1,39 \\
Pupa & 1,78 & 0,42 \\
Adulto: & & \\
Hembra & 15,93 & 6,93 \\
Macho & 11,83 & 5,28 \\
\hline
\end{tabular}

FIGUAA 1
Ciclo de vida para el mosquito Aedes taeniorhynchus (hembra)

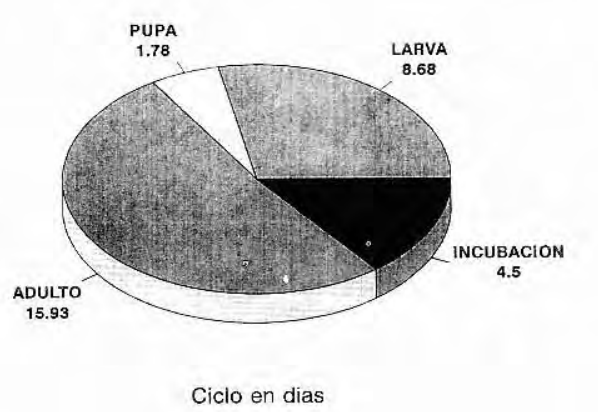

FGUFA 2
Ciclo de vida para el inosquito Aedes taeniorhynchus (macho)

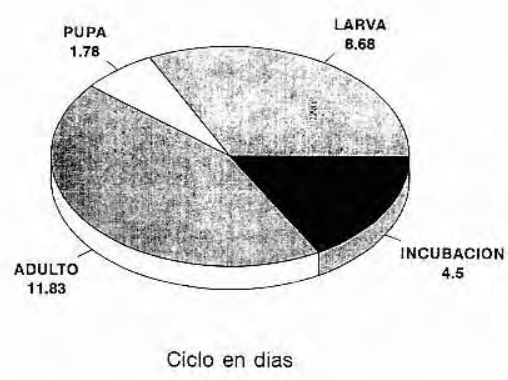

Curva de supervivencia para una cohorte de 100 individuos adultos machos y cien individuos aduitos hembras, del mosqulto Aedes laenlorhynchus en condiciones de laboratorio.

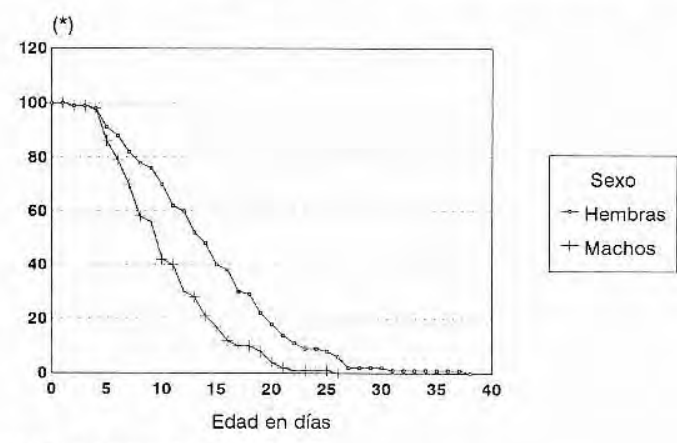

(9) PROPORCION DE SUPERVIVENTES

Se obtuvo oviposición normalmente en las tazas que contenían tierra humedecida; sin embargo, dió buenos resultados con cajas de Petri a las cuales se les colocó en el fondo algodón suficientemente humedecido.

Para la alimentación de las larvas se administró Ken-L, alimento para perros, previamente molido. Las hembras adultas se alimentaban de sangre de curí. A adultos machos y hembras se les mantenía permanentemente la solución azucarada.

Las condiciones físicas de laboratorio estandarizadas fueron en promedio: $27^{\circ} \mathrm{C}$ de temperatura, $85 \%$ de humedad relativa y 12 horas luz. 
Tabla 3. Tabla de vida para una cohorte de 100 individuos adultos machos del mosquito Aedes taeniorhynchus en condiciones de laboratorio.

\begin{tabular}{|c|c|c|c|c|c|c|}
\hline$X$ dias & $q x$ & $1 x$ & $d x$ & Lx & Tx & Ex \\
\hline 0 & 0,000 & 100 & 0 & 100 & 1128 & 11,28 \\
\hline 1 & 0,000 & 100 & 0 & 100 & 1028 & 10,28 \\
\hline 2 & 0,010 & 100 & 1 & 99 & 928 & 9,28 \\
\hline 3 & 0,010 & 99. & 1 & 98 & 829 & 8,37 \\
\hline 4 & 0,051 & 98 & 5 & 95 & 731 & 7,45 \\
\hline 5 & 0,118 & 93 & 11 & 87 & 636 & 6,83 \\
\hline 6 & 0,097 & 82 & 8 & 78 & 549 & 6,69 \\
\hline 7 & 0,081 & 74 & 6 & 71 & 471 & 6,36 \\
\hline 8 & 0,176 & 68 & 12 & 62 & 400 & 5,88 \\
\hline 9 & 0,035 & 56 & 2 & 55 & 338 & 6,03 \\
\hline 10 & 0,166 & 54 & 9 & 49 & 283 & 5,24 \\
\hline 11 & 0,088 & 45 & 4 & 43 & 234 & 5,20 \\
\hline 12 & 0,121 & 41 & 5 & 38 & 191 & 4,65 \\
\hline 13 & 0,111 & 36 & 4 & 34 & 153 & 4,25 \\
\hline 14 & 0,187 & 32 & 6 & 29 & 119 & 3,71 \\
\hline 15 & 0,192 & 26 & 5 & 23 & 90 & 3,46 \\
\hline 16 & 0,285 & 21 & 6 & 18 & 67 & 3,19 \\
\hline 17 & 0,266 & 15 & 4 & 13 & 49 & 3,26 \\
\hline 18 & 0,000 & 11 & 0 & 11 & 36 & 3,27 \\
\hline 19 & 0,272 & 11 & 3 & 9 & 25 & 2,27 \\
\hline 20 & 0,250 & 8 & 2 & 7 & 16 & 2,00 \\
\hline 21 & 0,333 & 6 & 2 & 5 & 9 & 1,50 \\
\hline 22 & 0,750 & 4 & 3 & 2 & 4 & 1,00 \\
\hline 23 & 0,000 & 1 & 0 & 1 & 2 & 2,00 \\
\hline 24 & 0,000 & 1 & 0 & 1 & 1 & 1,00 \\
\hline 25 & 1,000 & 1 & 1 & 0 & 0 & 0,00 \\
\hline 26 & 0 & - & - & - & - & - \\
\hline
\end{tabular}

qx: porcentaje de la cohorte muerto

1x: número de individuos de la cohorte inicial

dx: número de individuos muertos durante cada intervalo de tiempo

Lx: número total de unidades de tiempo

Tx: número de unidades de tiempo vividas por la cohorte

Ex: esperanza de vida 
Tabla 4. Tabla de vida para una cohorte de 100 individuos adultos hembras del mosquito Aedes taeniorhynchus en condiciones de laboratorio.

\begin{tabular}{|c|c|c|c|c|c|c|}
\hline$X$ días & $q x$ & $1 x$ & $d x$ & $L x$ & $T x$ & Ex \\
\hline 0 & 0,000 & 100 & 0 & 100 & 1538 & 15,38 \\
\hline 1 & 0,000 & 100 & 0 & 100 & 1438 & 14,38 \\
\hline 2 & 0,010 & 100 & 1 & 99 & 1338 & 13,38 \\
\hline 3 & 0,010 & 99 & 1 & 98 & 1239 & 12,51 \\
\hline 4 & 0,030 & 98 & 3 & 96 & 1141 & 11,64 \\
\hline 5 & 0,031 & 95 & 3 & 93 & 1045 & 11,00 \\
\hline 6 & 0,032 & 92 & 3 & 90 & 952 & 10,34 \\
\hline 7 & 0,056 & 89 & 5 & 86 & 862 & 9,68 \\
\hline 8 & 0,047 & 84 & 4 & 82 & 776 & 9,23 \\
\hline 9 & 0,025 & 80 & 2 & 79 & 694 & 8,67 \\
\hline 10 & 0,102 & 78 & 8 & 74 & 615 & 7,88 \\
\hline 11 & 0,057 & 70 & 4 & 68 & 541 & 7,72 \\
\hline 12 & 0,075 & 66 & 5 & 63 & 473 & 7,16 \\
\hline 13 & 0,114 & 61 & 7 & 57 & 410 & 6,72 \\
\hline 14 & 0,055 & 54 & 3 & 52 & 353 & 6,53 \\
\hline 15 & 0,098 & 51 & 5 & 48 & 301 & 5,90 \\
\hline 16 & 0,130 & 46 & 6 & 43 & 253 & 5,50 \\
\hline 17 & 0,175 & 40 & 7 & 38 & 210 & 5,25 \\
\hline 18 & 0,060 & 33 & 2 & 32 & 172 & 5,21 \\
\hline 19 & 0,193 & 31 & 6 & 28 & 140 & 4,51 \\
\hline 20 & 0,080 & 25 & 2 & 24 & 112 & 4,48 \\
\hline 21 & 0,260 & 23 & 6 & 20 & 88 & 3,82 \\
\hline 22 & 0,352 & 17 & 6 & 14 & 68 & 4,00 \\
\hline 23 & 0,000 & 11 & 0 & 11 & 54 & 4,90 \\
\hline 24 & 0,181 & 11 & 2 & 10 & 43 & 3,90 \\
\hline 25 & 0,000 & 9 & 0 & 9 & 33 & 3,66 \\
\hline 26 & 0,444 & 9 & 4 & 7 & 24 & 2,66 \\
\hline 27 & 0,600 & 5 & 3 & 3 & 17 & 3,40 \\
\hline 28 & 0,000 & 2 & 0 & 2 & 14 & 7,00 \\
\hline 29 & 0,000 & 2 & 0 & 2 & 12 & 6,00 \\
\hline 30 & 0,000 & 2 & 0 & 2 & 10 & 5,00 \\
\hline 31 & 0,000 & 2 & 0 & 2 & 8 & 4,00 \\
\hline 32 & 0,500 & 2 & 1 & 1 & 6 & 3,00 \\
\hline 33 & 0,000 & 1 & 0 & 1 & 5 & 5,00 \\
\hline 34 & 0,000 & 1 & 0 & 1 & 4 & 4,00 \\
\hline 35 & 0,000 & 1 & 0 & 1 & 3 & 3,00 \\
\hline 36 & 0,000 & 1 & 0 & 1 & 2 & 2,00 \\
\hline 37 & 0,000 & 1 & 0 & 1 & 1 & 1,00 \\
\hline 38 & 1,000 & 1 & 1 & 0 & 0 & 0,00 \\
\hline 39 & 0 & - & - & - & - & - \\
\hline
\end{tabular}




\section{Discusión}

Al confrontar el número promedio de adultos que emergen de las pupas en las generaciones estudiadas con las no viables, se pudo concluir la gran eficiencia de la colonia, como resultado de la relativa facilidad de adaptación de la especie a las condiciones ambientales, físicas y nutricionales a nivel de laboratorio.

Al inundar las tazas con los huevos embrionados, se registró el tiempo promedio de eclosión de las larvas en todas las generaciones; sin embargo, hubo diferencias significativas en la quinta generación (5,33 minutos); esto es explicable en la medida en que las condiciones de humedad de la tierra contenida en las tazas eran mucho mejores que en las demás generaciones y posiblemente se mantuvo estable durante todo el tiempo de incubación de los huevos, e igualmente se vió favorecida por la temperatura más alta del agua contenida en las cápsulas de porcelana en donde se introdujeron las tazas para ser inundadas.

Al observar y registrar el ciclo de vida del mosquito en cada una de las generaciones, motivo de este estudio, se obtuvieron tiempos promedio definidos en días, para las diferentes etapas del desarrollo; aunque es claro que algunos factores ambientales y nutricionales pueden aumentar o reducir este tiempo. De mención particular es el caso de las larvas que al adicionarles una mayor cantidad de alimento (sin ser excesivo), produjo un crecimiento relativamente acelerado $\mathrm{y}$, por tanto, reducción del tiempo empleado en su desarrollo. Contrariamente, el hacinamiento de las larvas en las bandejas, les provocaba una disminución en el tamaño y, por ende, a las generaciones de pupas y adultos.

Cuando se utilizan los viales como sitio de permanencia para los mosquitos adultos hembras, se registra un porcentaje mayor de supervivencia; esto se debe a la influencia de algunos factores ecológicos, tales como la no competencia por el alimento y a un gasto mínimo de energía; además, dentro del vial se da un microhábitat donde la temperatura es más constante y la humedad relativa es alta.
Contrario a lo informado por Forattinni en 1965 (3), el mínimo cambio de agúa en las bandejas (dos veces) ocasiona menor mortalidad a las larvas.

Los adultos dentro de la jaula prefieren como lugar de reposo las esquinas, la chupa y el exterior e interior de las tazas plásticas con tierra, absteniéndose de posarse en el anjeo de las mismas; por tanto, el espacio donde habitan y circulan se reduce; esto origina mayor gasto de energía al permanecer los mosquitos más tiempo volando para encontrar un espacio libre y apropiado donde asentarse; en consecuencia, un alto número de adultos (hacinamiento) en una jaula, causa gran índice de mortalidad y, a la inversa, prolonga su longevidad.

La mayor longevidad de las hembras, eficientes vectores del arbovirus, favorece la replicación del virus en los diferentes tejidos del mosquito. Los arbovirus necesitan un período de incubación para poder desarrollarse en varios tejidos y lograr que éstos alcancen las glándulas salivales.

\section{Agradecimientos}

Los autores desean expresar sus sinceros agradecimientos a COLCIENCIAS, la Universidad de La Salle, en cooperación con el Instituto Nacional de Salud de Colombia, por la financiación y apoyo brindados al presente trabajo.

A los doctores Abraham Hadra S. y Julio Guzmán, Director del Departamento de Bioquímica y de la Oficina de Investigaciones de la Universidad de La Salle, respectivamente, por la colaboración prestada.

Al señor Gilberto Torres por la asistencia brindada en el mantenimiento de la colonia.

\section{Referencias}

1. Beeson MPB, McDeermott W. Tratado de medicina interna. México: Interamericana, 1977:1-1039.

2. Curso de control de vectores. Conservación y montaje de mosquitos. Universidad de Carolina del Sur, E.U.A. $1984 ; 1$

3. Forattinni OP. Entomología médica. Sao Paulo: Universidad de Sao Paulo. 1965:2-662. 
4. Galán M, Bisset JA, Marquetti M, et al. Algunas observaciones del comportamiento de Aedes taeniorhynchus (Wiedemann, 1821) y Aedes solicitans (Walkers, 1956). Rev Cuba Med Trop 1986; 38:69.

5. Gerberg EJ. Manual for mosquito rearing and experimental techniques. American Mosquito Central Association Bulletin 5, 1970.

6. Groot H. The health and economic impact of Venezuelan Equine Encephalitis (V.E.E.).In:Venezuelan Encephalitis. Washington, D.C.:PAHO. 1972: 7.
7. Nielsenl LT. Mosquitos systematics. UTAH: American Mosquito Control Association 1983; 15:162.

8. Olano VA. Hallazgo de Aedes taeniorhynchus (Wiedemann, 1821) en un lugar del municipio de Ambalema departamento del Tolima (Colombia) (Dipteria: Culicidae). $1985 ; 5:(1-2) 26$.

9. Poole RW. An introduction to quantitative ecology. New York: McGraw-Hill 1974; 11.

10. Tinker ME. Clave práctica para los mosquitos de recipiente en Colombia. Mimeografiado 1981; 1. 